\title{
VEHICLE NUMBER-PLATE LOCALIZATION AND RECOGNITION USING ENERGY BASED FEATURES
}

\author{
B. RaviKiran ${ }^{1}$ and Shaik. Matheen Basha ${ }^{2}$
}

\begin{abstract}
Automatic vehicle number-plate recognition has been a challenging task in the field of computer vision. It has significant importance in various security and traffic applications. Several recognition schemes in this regard have been proposed by researchers. In this work, a novel and efficient scheme is proposed for automatic recognition of vehicle number-plates. The scheme comprises of two different phases. The first phase takes care of localization and segmentation of the number-plate in a scene image. For this, suitable wavelet coefficients are considered. The subsequent phase takes care of the recognition task. For the purpose, template matching concept has been utilized. During experimentation, a total of 300 numberplate images are taken as input. Plate localization accuracy of $96 \%$ and recognition rate of $94.5 \%$ are obtained satisfactorily.
\end{abstract}

\section{INTRODUCTION}

The vehicle-plate recognition system (VPRS) generally consists of three distinct parts: (a) detection of appropriate only number-plate (NP) (commonly known as localization); (b) Extraction of the character images only from the NP (commonly known as segmentation); and (c) recognition by classification. Several state-of-the-art techniques have been presented in the literature in this context. Few of the commercial products have also been released, although, they have their own limiting constraints like the proper lighting, noise parameter, resolution of the image captured, computation time, and other environment related factors. Development of an efficient recognition scheme has still been a challenging task subject to these constraints.

Various approaches as reported in the literature for vehicle number-plate recognition have been presented below.

The methods based on textures mainly exploited the aspect ratio [1], [2], [3], the contrast variations [4], the uniform distribution of the characters [5], and the ratio between background area and character area [6], [7]. Among these, the approaches in [1] and [3] are applied to the LPRS in Saudi Arabia. The main idea is to process the image as a grayscale image and employ

${ }^{1}$ Department of CSE, ANITS, Visakhapatnam

${ }^{2}$ Department of EEE, GVP college of Engineering, Visakhapatnam 
the Sobel edge detection, projection, and seed-filling algorithm [8] to remove the regions unrelated to the LP. The result is then filtered by the aspect ratio and object connections. However, the method is not capable of dealing with the complex environment and rotation. Nonetheless, the rotation problem can be eased by considering the rotation extent [2]. Hsieh et al. [5] employed the Wavelet transform to decompose the image into four bands (i.e., HH, HL, LH, and LL). The searching range of the LP is restricted according to the image property in the LH band to improve the processing speed. The projection method, edge density, and aspect ratio are then exploited to locate the LP. This approach is proven to be effective in withstanding the variation of the lighting, contrast, and rotation and is also capable of locating multiple plates. $\mathrm{Wu}$ et al. [7] adopted the morphological projection to localize the LP. The method exploited the ratio between background area and character area and used the opening operation to blur the image. The processed image is compared to the original image. The discrepancy part is then processed with the projection method to locate the LP. The advantage of this method is high efficiency, whereas the disadvantage is easily undergone by the interference of the lighting effect. The methods based on the colors are discussed as follows. Jia et al. [6] adopted the mean shift to blur the image and then localized the LP by applying the Mahalanobis distance linear classifier to classify the candidate regions according to rectangularity, aspect ratio, and edge density. The object connection is applied to the classified regions separated by the edges and further locates the LP. Chang et al. [10] converted the red-green-blue (RGB) color space into the huesaturation-intensity (HSI) model and then performed the blurring process to reduce the interference of noise. The clustered edge property of the LP is exploited to cooperate with the HSI color spaces to locate the LP. The advantage of this method is the capability of withstanding the interference of the environment.

\section{PROPOSED SCHEME}

The proposed system comprises of three stages namely, image acquisition, localization of vehicle number-plate, and character recognition there in. A block diagram of the proposed system is provided in Fig.1. All the stages of the proposed system are explained below in a sequence.

\section{(I) Image Acquisition:}

In the first phase, we capture the image of the vehicle and normalize to a standard dimension of $400 \times 300$ pixels. We then convert the RGB image into a grayscale one $\left(I_{g g}\right)$ using the equation (eq.1) as given below:

$I_{g z}=\frac{3 * I_{r a d}+6 m I_{\text {gran }}+I_{b l u z}}{10}$

where, $I_{\text {red }}, I_{\text {green }}$, and $I_{\text {bine }}$ are the color spectrums of the image along the red, green, and blue channels respectively.

\section{(II) Localization of the plate:}

The image $I_{g z}$ is considered as the candidate for plate localization and wavelet decomposition applied to compute the approximation and detail coefficient matrices. Fig.2 shows the four frequency bands representing low, horizontal, vertical, and diagonal frequency energy, respectively. The horizontal and vertical frequency energies can locate the license plate because 
the plate has a high frequency, so to find the plate's vertical location; we plot the pixel intensity's energy curve for each row of the vertical frequency band. Similarly, to find the plate's horizontal location, we plot the pixel intensity's energy curve for each column of the horizontal frequency band. Fig. 3 shows the plots.
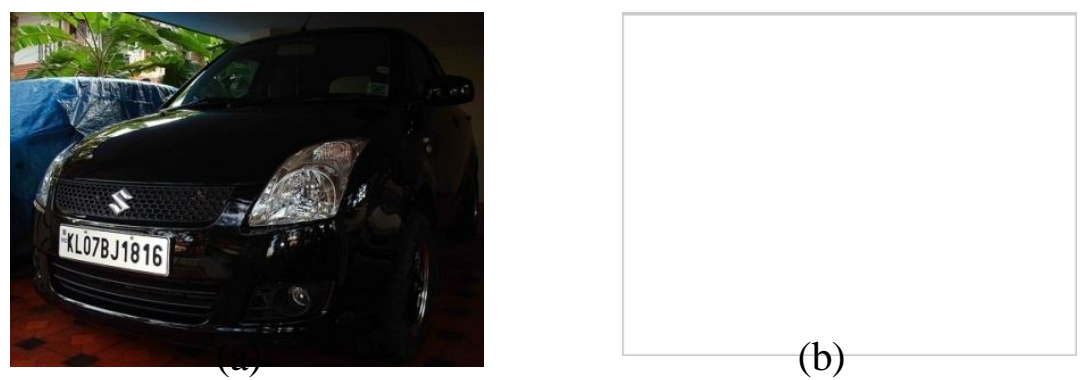

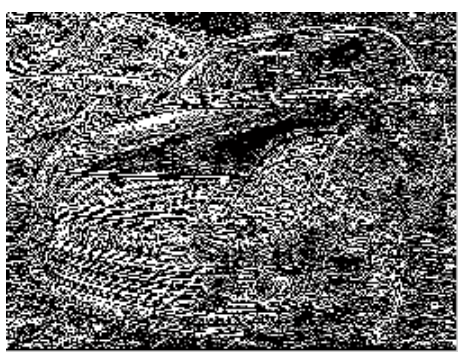

(c)

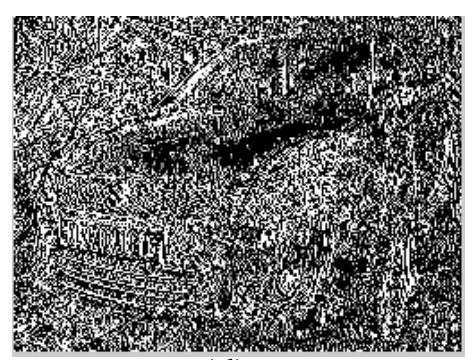

(d)

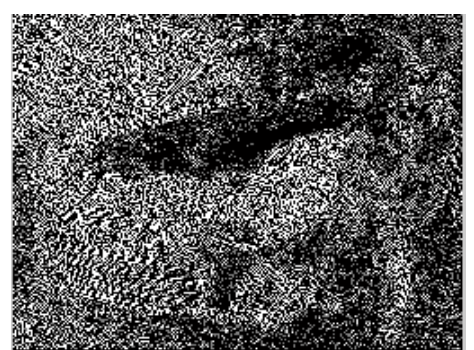

(e)

(Fig.2: (a)-Sample input image; (b)-' ca' component; (c)-' ch' component; (d)-' cv' component; and (d)-cd component after decomposition of the image.)
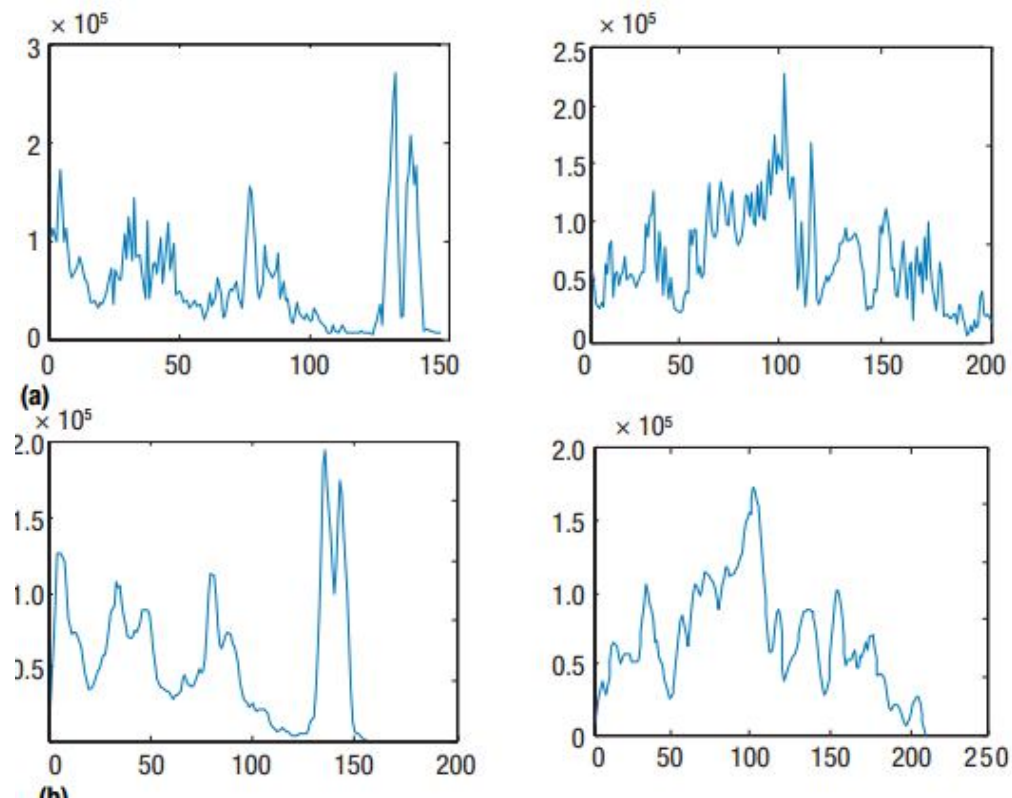

(Fig.3: Plot of the vertical (a) and horizontal (b) frequency bands for the input sample image.) 
A Gaussian filter modifies the input signal with a Gaussian function. Equation (eq.2) gives the one-dimensional impulse response:

$h(x)=\sqrt{\frac{a}{\pi}} e^{-a x^{2}}$

This eq. 2 gives the frequency response as:

$h^{\prime}(f)=e^{-\frac{\pi^{n} f}{a}}$

where, $f$ is the ordinary frequency. These equations can also be expressed with the standard deviation as a parameter:

$h(x)=\frac{1}{\sqrt{2 \pi \sigma}} e^{-\frac{x^{2}}{\sigma^{2}}}$

Finally, the frequency response will be:

$h^{\prime}(f)=e^{-\frac{\pi^{2} f^{2}}{2 \sigma^{2} f}}$

There are several advantages of using Gaussian filter: it is rotationally symmetric; filter weights decrease monotonically from the central peak, giving the most weight to central pixels; there is a simple and intuitive relationship between the size of $\sigma$ and the smoothing parameter; and Gaussian filtering is also separable. The later aspect provides extra feature: becoming separable means that it first does the convolution of the image with a 1-D horizontal filter and later does the convolution of the result with a 1-D vertical filter. For a $k \times$ Gaussian filter, 2-D convolution requires $\mathrm{k}^{2}$ operations for each pixel, whereas, by using the separable filters, we reduce this to 2 $\mathrm{x} k$ operations for each pixel. Fig. 2 shows the results of applying the Gaussian filter on both the energy curves. The high energy of the vertical curve represents the required vehicle numberplate. This is because, as we mentioned earlier, we find a higher frequency around the numberplate. For computing the high-energy frequency band, a threshold value is determined to operate on the vertical and horizontal frequency energy curves individually. We take half the maximum vertical energy and traverse from left to right and right to left in the vertical frequency curve to compute the $y$ coordinates. The $y$ coordinates that are greater than the threshold value represent the plate's vertical location. Figure 3 demonstrates an example for this.

To accurately determine the horizontal location, rather than to just operate on the horizontal frequency energy curve in Fig.2, the same operation is performed on the image in Fig.3. If we traverse from left to right on this image, it is clearly observed that the frequency is more near the number plate, so to find the horizontal location of the number-plate, we repeat the process we did to find it vertically. Fig.4 shows the result. It is to be noted that we do not need half the maximum of the $y$ coordinates to calculate the threshold value. This is because the frequency is very high near the number plate compared to other locations. Instead, we take 0.9 times the maximum value to compute the threshold value and traverse from left to right and right to left to compute the $x$ coordinates. Fig.5 shows the result. 

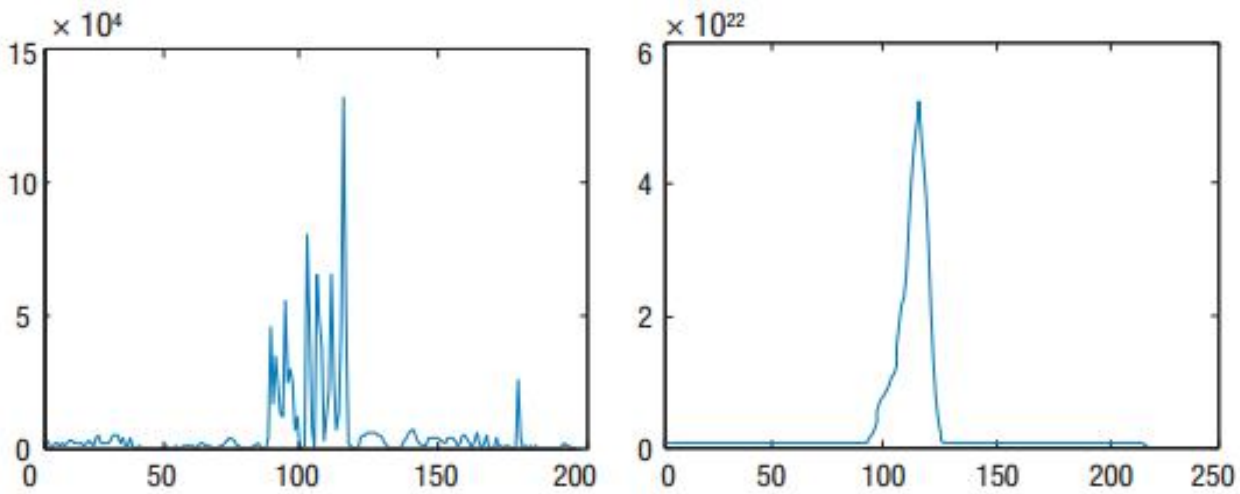

(Fig.4: Deterpination of peak through thresholding by $90 \%$ (b)

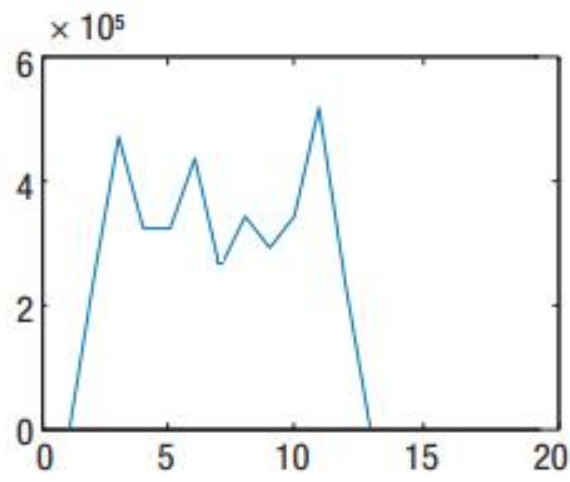

\section{KLO7BJ9816 KL07B)1816}

(Fig.5: Extraction of the ROI and recognition output.)

\section{(III) Character Recognition:}

After the completion of the segmentation process, the individual character images are standardized to a uniform size of 64 X 64 (pixels). For matching these character images against the database this standardization process is needed as the database also contains objects of identical dimension, i.e. $64 \times 64$ (pixels). The subsequent phase is template matching that should be an efficient matching algorithm. Template matching compares these segmented individual character images with the those in the database for getting the best match. We use a statistical correlation-based method to measure the correlation coefficient between the probe and template images, where the highest correlation represents the best match. We use a database of 36 alphanumeric characters (26 letters in capital only and 10 numbers. Despite good recognition rate, few misclassification are noticed due to some structural similarities as found in case of ' $\mathrm{O}^{\prime}$ and ' $\mathrm{O}^{\prime}$. To compensate for this error, the aspect ratio is considered as an extra parameter during the matching phase which dramatically resolved misclassification the problem. 


\section{EXPERIMENTAL EVALUATION}

For the performance evaluation of the proposed scheme, we performed experiments on ten different models of cars with different shapes, sizes, and colors of plates under varying lighting conditions and distances. Size of the input images is taken to be of dimension $128 \times 128$ pixels. A total of 300 such vehicle number-plates are considered for the purpose. Table 1 depicts the experimental results. Commendable results are achieved by the proposed scheme. The rate of accuracy for the number-plate localization and for the rate of character recognition is found to be 96\% and $94.5 \%$ respectively. In Table 2, comparative analysis of the proposed scheme with other state-of-the-art schemes has been provided. It is evident from this table that the proposed scheme outperforms others.

Table 1: Result analysis of the proposed scheme.

\begin{tabular}{|l|l|l|}
\hline Criteria & Sample size & Result \\
\hline Number-plate localization & Number of samples & Accuracy (\%) \\
\hline Correctly localized: & 288 & 96 \\
\hline Wrongly localized: & 12 & ---- \\
\hline & ---- & ---- \\
\hline Individual plate segmentation & ---- & ---- \\
\hline Total number of character images: & 2592 & ---- \\
\hline Correctly segmented: & 2514 & 97 \\
\hline & ---- & ---- \\
\hline Character recognition & ---- & ---- \\
\hline Total character images: & 2514 & ---- \\
\hline Correctly recognized: & 2375 & 94.5 \\
\hline Misclassification: & 139 & ---- \\
\hline & & \\
\hline
\end{tabular}

Table 2: Performance comparison.

\begin{tabular}{|l|l|l|l|}
\hline Scheme & Rate of localization & $\begin{array}{l}\text { Rate of } \\
\text { segmentation }\end{array}$ & Rate of recognition \\
\hline Chiou et. al & 93.2 & ---- & 96.2 \\
\hline Wang et. al & 94 & ---- & 94 \\
\hline Kang et. al & ---- & 86.5 & 86.5 \\
\hline $\begin{array}{l}\text { Proposed } \\
\text { scheme }\end{array}$ & 96 & 97 & 94.5 \\
\hline
\end{tabular}

\section{CONCLUSIONS:}

An efficient scheme for automatic vehicle number-plate recognition has been proposed. The scheme emphasizes on three different aspects namely, localization of the number-plate in the image, segmentation of individual character images from the region of interest, and proper recognition of the individual characters. The scheme has been tested and validated on suitable database. High rates of localization, segmentation, and recognition has been achieved upon successful implementation of the scheme. It is also compared against state-of-the-art techniques and it is found to be outperforming those in every respect. 


\section{REFERENCES:}

[1] M. Sarfraz, M. J. Ahmed, and S. A. Ghazi, "Saudi Arabian license plate recognition system," in Proc. IEEE Int. Conf. GMAG, 2003, pp. 36-41.

[2] M. Yu and Y. D. Kim, "An approach to Korean license plate recognition based on vertical edge matching," in Proc. IEEE Int. Conf. Syst., Man, Cybern., 2000, vol. 4, pp. 2975-2980.

[3] M. J. Ahmed, M. Sarfaz, A. Zidouri, and K. G. AI-Khatib, "License plate recognition system," in Proc. IEEE ICECS, 2003, pp. 898-901.

[4] T. H. Wang, F. C. Ni, K. T. Li, and Y. P. Chen, "Robust license plate recognition based on dynamic projection warping," in Proc. IEEE Int. Conf. Netw., Sensing Control, 2004, pp. 284-288.

[5] C. T. Hsieh, Y. S. Juan, and K. M. Hung, "Multiple license plate detection for complex background," in Proc. IEEE 19th Int. Conf. AINA, 2005, pp. 389-392.

[6] W. Jia, H. Zhang, and H. Xiangjian, "Mean shift for accurate number plate detection," in Proc. IEEE 3rd ICITA, 2005, pp. 732-737.

[7] C. Wu, L. C. On, C. H. Weng, T. S. Kuan, and K. Ng, "A Macao license plate recognition system," in Proc. IEEE 4th Int. Conf. Mach. Learn. Cybern., Guangzhou, China, 2005, pp. 4506-4510.

[8] A. R. Smith, “Tint fill,” Comput. Graph., vol. 13, no. 2, pp. 276-283, Aug. 1979.

[9] S. Wesolkowski and E. Jernigan, "Color edge detection using jointly Euclidean and vector angle," in Proc. Vis. Interface, May 1999, pp. 19-21.

[10] S. L. Chang, L. S. Chen, Y. C. Chung, and S. W. Chen, "Automatic license plate recognition,” IEEE Trans. Intell. Transp. Syst., vol. 5, no. 1, pp. 42-53, Mar. 2004. 